\title{
Subcutaneous formulation of belimumab in treatment of systemic lupus erythematosus: a critical review with focus on safety and satisfaction
}

This article was published in the following Dove Press journal:

Patient Preference and Adherence

\author{
Hamdy MA Ahmed ${ }^{1-3}$ \\ Samar Abohamad ${ }^{2}$ \\ Mohanad Elfishawi ${ }^{4}$ \\ Mohamed Tharwat Hegazy ${ }^{2}$ \\ Kadambari Vijaykumar ${ }^{1,5}$ \\ 'Internal Medicine Department, \\ Rochester General Hospital, \\ Rochester, NY, USA; ${ }^{2}$ Rheumatology \\ and Clinical Immunology Unit, \\ Internal Medicine Department, \\ Faculty of Medicine, Cairo University, \\ Cairo, Egypt; ${ }^{3}$ Rheumatology and \\ Clinical Immunology Division, \\ Internal Medicine Department, \\ University of Alabama at Birmingham, \\ Birmingham, AL, USA; ${ }^{4}$ Internal \\ Medicine Department, Queens \\ Hospital Center, Icahn School of \\ Medicine at Mount Sinai, New York, \\ NY, USA; ${ }^{5}$ Pulmonology and Critical \\ Care Division, Internal Medicine \\ Department, University of Alabama at \\ Birmingham, Birmingham, AL, USA
}

Correspondence: Hamdy MA Ahmed Internal Medicine Department, Rochester General Hospital, 1425 Portland Avenue,

Rochester, NY I462I, USA

Tel +I 5859224829

Fax + I 5859224440

Email dr.hamdy86@gmail.com

\begin{abstract}
Belimumab is a novel add-on therapy that has been approved for patients with active and antibody-mediated systemic lupus erythematosus. It is a monoclonal antibody that decreases the activation of B-cells and consequently decreases antibodies' production. Recently, the US Food and Drug Administration approved subcutaneous belimumab for patients who have received training on using it. Subcutaneous belimumab can be administered using either a prefilled syringe or an auto-injector device. Weekly subcutaneous belimumab seems to be as effective as monthly intravenous belimumab with a similar safety margin. In this article, we reviewed the literature on subcutaneous belimumab focusing on safety and patients' experiences and satisfaction. Overall, subcutaneous belimumab appears to be preferred over intravenous belimumab for a number of reasons. However, more studies are still required to prove these findings.
\end{abstract}

Keywords: belimumab, subcutaneous, safety, self-injection, patient satisfaction, adherence, auto-injector

\section{Introduction}

Systemic lupus erythematosus (SLE) is a chronic systemic autoimmune disease associated with variable clinical presentations. Its prevalence in the US is estimated to be up to 150 patients per $100,000 .^{1}$ The disease typically runs a relapsing course and can result in multiple co-morbidities. ${ }^{2}$ Mortality may occur early in the course of the disease. Causes of mortality and morbidity include severe organ damage, infections as a result of suppressed immune system, and cardiovascular events secondary to accelerated atherosclerosis. ${ }^{3}$ The precise etiology of SLE is not known and is postulated to be secondary to genetic, environmental, immune, and/or hormonal factors. However, despite the unclear etiology, it is known that most of the disease manifestations are mediated by the formation of different autoantibodies and antigen-antibody complexes. ${ }^{48}$ This abundance of autoantibodies in SLE is reflective of a significant role of B-cell-mediated pathogenesis. ${ }^{9}$

Earlier guidelines for SLE treatment recommended the use of antimalarial medications, nonsteroidal anti-inflammatory drugs, and corticosteroids. ${ }^{10,11}$ Later, immunosuppressant medications were recommended for specific systems' involvement, severe disease, and as corticosteroid sparing agents. ${ }^{12-14}$ Since 1998 , hydroxychloroquine has been the only medication approved by the US Food and Drug Administration (FDA) for the treatment of SLE. ${ }^{15,16}$ In 2011, the FDA approved the use of belimumab for treatment of patients with antibody-positive active SLE without active lupus nephritis or central nervous system (CNS) involvement. In July 2017, the FDA approved the use 
of the subcutaneous (SC) form of belimumab by patients who received user training from their health care providers. ${ }^{17}$

In this review, we used different combinations of keywords "Belimumab", "Subcutaneous", "Preference", "Safety", and "Efficacy" in PubMed, Cochrane, and Google Scholar to find studies and articles that discussed efficacy, safety, and patient preference of SC belimumab.

\section{Belimumab}

BAFF protein, known as B-cell lymphocyte stimulator, is a TNF that binds to different receptors in the B-cell resulting in proliferation, differentiation, and secretion of antibodies. ${ }^{18,19}$ Elevated levels of BAFF have been found in patients with SLE and have been linked to disease flares. ${ }^{20-23}$ Belimumab is a human monoclonal IgG1 antibody that binds to BAFF protein resulting in a significant decrease in the number and activation of B-cells with a sequential decrease in autoantibodies' production. ${ }^{24,25}$ Clinical trials have demonstrated that belimumab resulted in significant reduction of anti-dsDNA (double-stranded DNA) antibody, improvement of complement levels, and resolution of hypergammaglobulinemia. ${ }^{26-29}$

\section{SC belimumab}

After the successful use of intravenous (IV) belimumab in the treatment of SLE, ${ }^{30-35}$ the need for more convenient routes of administration, especially SC injection, has emerged. ${ }^{36}$ Phase I studies demonstrated an acceptable bioavailability with different doses of SC belimumab. Moreover, weekly SC belimumab dosed at $200 \mathrm{mg}$ achieved blood levels and bioavailability similar to those achieved by a monthly $10 \mathrm{mg} / \mathrm{kg}$ IV belimumab dose, regardless of the site of injection or body size. SC belimumab achieved maximum serum concentration within 3.9-5.9 days with a half-life of 18.2 days. ${ }^{37-39}$ Phase II studies indicated an acceptable safety profile of SC belimumab similar to the IV form. ${ }^{40-42}$

In a 52-week double-blinded, randomized controlled Phase III study (BLISS-SC), SC belimumab added to standard of care showed significantly higher efficacy, using SLE response index (SRI-4), time to severe flare, and corticosteroid dose reduction, compared to standard of care plus placebo. ${ }^{43}$ Similar to IV belimumab, its efficacy was higher in a difficult-to-treat group of patients with positive antidsDNA and low complement level. ${ }^{44}$ Moreover, in a 24-week open-label extension study that included 662 patients, SC belimumab continued to show the same efficacy. ${ }^{45}$ Efficacy of SC belimumab was not different from IV belimumab when both were indirectly compared. ${ }^{46}$ It is important to remember that only SLE patients with moderate to severe disease (score of $\geq 8$ on the Safety of Estrogens in Lupus Erythematosus National Assessment version of the SLE Disease Activity Index) were included in all previously mentioned studies. ${ }^{32,33,35,43}$

\section{Forms of SC belimumab}

Currently, SC belimumab can be administered via either autoinjector or prefilled syringes. Both devices contain a single dose of $1 \mathrm{~mL}$ of preservative-free liquid solution (polysorbate 80 , L-arginine hydrochloride, L-histidine, L-histidine monohydrochloride, and sodium chloride) in which $200 \mathrm{mg}$ of belimumab is dissolved. Both devices come with a fixed 27-gage, half inch needle and are latex free.

$\mathrm{SC}$ belimumab should be stored at a temperature of $2^{\circ} \mathrm{C}-8^{\circ} \mathrm{C}\left(36-46^{\circ} \mathrm{F}\right)$. It should be allowed to warm at room temperature for 30 minutes before administration. SC tissue of both thighs and the abdomen (except for $2 \mathrm{~cm}$ around the umbilicus) are recommended as injection sites. ${ }^{47}$

\section{Safety of SC belimumab}

In the 52-week BLISS-SC study, safety results in the belimumab group (556 patients) were comparable to the safety results in the placebo group (280 patients). Total adverse events were reported in $84 \%$ vs $80 \%$ in the placebo and the belimumab groups, respectively. Most common adverse events reported were infections. Serious adverse events were, surprisingly, reported more in the placebo group (15.7\% vs $10.8 \%)$. As expected, treatment-related adverse events were higher in the belimumab group ( $31 \%$ vs $26 \%$ ). Study discontinuation, due to adverse events, was more in the placebo group ( $8.9 \%$ vs $7.2 \%$ ). Herpes zoster was reported more in the placebo group ( $4.6 \%$ vs $3.2 \%)$. There were three $(0.5 \%)$ deaths in the belimumab group that resulted from bacterial sepsis, urosepsis, and CNS tuberculosis, and two (0.7\%) deaths in the placebo group that were secondary to cardiac arrest and SLE-related thrombocytopenia.

Although local site injection reactions were reported more in the belimumab group, post-injection hypersensitivity reactions were reported more in the placebo group. It was noted that the adverse events, in general, were higher in patients with higher body weight in both groups but this finding was not explained. Depression was reported more in the placebo group (3.6\% vs $2.7 \%)$. Suicidal ideation, with no execution/ fatality, was reported in $0.4 \%$ of the belimumab patients. ${ }^{43}$

Safety results continued to be similar in the group of patients with positive anti-dsDNA and low complement level. Total and treatment-related adverse events rates were similar 
to previously mentioned results. However, serious adverse events were higher in both treatment and placebo groups compared to the previously mentioned study, and continued to be higher in the placebo group (23.1\% vs $13.3 \%){ }^{44}$

Even after a 24-week extension of treatment, SC belimumab continued to be well tolerated and showed similar adverse events rates with lower treatment discontinuation rate. ${ }^{45} \mathrm{SC}$ belimumab resulted in less adverse events than IV belimumab; however, this was not statistically significant. ${ }^{46}$

\section{Patient satisfaction with SC belimumab using the auto-injector device}

A two-part survey study evaluated the satisfaction of rheumatologists $(\mathrm{N}=88)$ and patients with SLE receiving treatment with and without belimumab (total $\mathrm{N}=513$ ). It concluded that patient satisfaction was mainly driven by their ability to function on a daily basis while physician satisfaction with treatment was driven primarily by different clinical outcomes. Patients and physicians strongly agreed that improvement of fatigue was a significant component of satisfaction with treatment. The authors suggested that belimumab could be considered in patients who report dissatisfaction with their standard of care.

More than half of the patients who received belimumab preferred to self-administer their injectable medications at home and considered the convenience and flexibility of treatment administration vital drivers for satisfaction. This preference was higher among patients who were employed. ${ }^{36}$

Another study that included 548 patients with SLE evaluated their preference for SC injection via an online survey. SC injection was the most preferred choice (41.2\%) followed by IV infusions (36.9\%). Surprisingly, $21.9 \%$ of the patients were not able to decide. Most patients in the latter group were the ones who did not have any previous experience with biological therapy. Older patients were more resistant to trying new SC injections. Patients who preferred SC injections emphasized the importance of not losing working days, avoiding going to the hospital, and being autonomous and comfortable at home. Those who expressed their preference for IV infusions emphasized the importance of feeling safe and the presence of qualified staff during therapy administration.

Despite the good number of study participants, it had some limitations as it relied only on patients who were able to use the internet and on their own interpretation of the questions and their knowledge. ${ }^{48}$
We found only one study that assessed the patients' experiences after using the auto-injector device for SC belimumab. ${ }^{49}$ Forty-three patients who received IV belimumab and SC belimumab via prefilled syringes were switched to SC belimumab via auto-injector device and were followed-up for 2 months. The authors used a questionnaire and a semi-structured telephone interview at the beginning and the end of the eight weeks. Approximately $88 \%$ of the patients were very satisfied, and $12 \%$ were satisfied with the training they received for using the auto-injector device. By the end of the study, $83 \%$ of the patients felt extremely confident using the auto-injector device correctly. Approximately $70 \%$ of the patients were very satisfied, $26 \%$ were satisfied, and 5\% were somewhat satisfied with using the auto-injector device.

The most common reported advantages of the autoinjector were convenience ( $71 \%$ ), easy and quick administration (57\%), and self-administration (24\%). By the end of the study, patients endorsed more advantages: shorter administration time (38\%), ease of incorporation into their daily routine (19\%), and ability to administer at home (19\%). On the other hand, $62 \%$ of the patients reported disadvantages including: injection-related discomfort, pain, stinging, nausea, and lack of control of injection speed. Pain was rated as mild in $83 \%$ of the patients, while $7 \%$ rated it as intermediate, and $10 \%$ rated it as severe. Other disadvantages included inconvenience which was explained by the need to store the medication in the refrigerator and the need to wait 30 minutes to achieve room temperature before using it.

Overall, $76 \%$ of the patients preferred the use of an autoinjector for SC belimumab over IV belimumab. Patients who preferred the IV form reported better control of their symptoms. In the same context, $52 \%$ reported no change in the severity of symptoms, while $24 \%$ reported improvement in symptoms that they thought was related to maintaining consistent levels of the medication with SC belimumab.

It is worth noting that the results from all previously mentioned studies could be biased as they relied on the memory recall of the patients included. Also, in such studies, patients may show higher compliance rates and their perspectives may be influenced by the fact that they are using new medication.

\section{Conclusion}

The majority of patients may prefer to use SC injections due to different reasons. Working status is a significant variable that may determine patients' preference to use SC injections. Health care staff should provide sufficient training on SC 
injections' administration and discuss their side effects and complications.

Data about SC belimumab were obtained from a limited number of studies. SC belimumab appears to be as effective as, and also as safe as, if not more so, than IV belimumab. $\mathrm{SC}$ belimumab is more convenient, time-saving, easier to use, and easier to incorporate into patients' daily routines. These advantages were appreciated more by patients who were employed. Disadvantages of SC belimumab include discomfort and pain at the injection site, storage in the refrigerator, and the need to wait for the injector to warm-up prior to use.

Further head-to-head studies comparing IV belimumab, SC belimumab using auto-injector and prefilled syringes in a larger sample size and with a longer follow-up duration are still needed to confirm the results of these preliminary data.

\section{Acknowledgment}

The authors would like to thank Bonita Archer, MLS, Cathey Carey, and Werner Medical Library staff for their help in reviewing the literature and gathering the articles used in this review.

\section{Disclosure}

The authors report no conflicts of interest in this work.

\section{References}

1. Lawrence RC, Hochberg MC, Kelsey JL, et al. Estimates of the prevalence of selected arthritic and musculoskeletal diseases in the United States. J Rheumatol. 1989;16(4):427-441.

2. Fernandez D, Kirou KA. What causes lupus flares? Curr Rheumatol Rep. 2016;18(3):14.

3. Cartella S, Cavazzana I, Ceribelli A, Inverardi F, Tincani A, Franceschini F. Evaluation of mortality, disease activity, treatment, clinical and immunological features of adult and late onset systemic Lupus erythematosus. Autoimmunity. 2013;46(6):363-368.

4. Cooper GS, Dooley MA, Treadwell EL, St Clair EW, Gilkeson GS. Risk factors for development of systemic lupus erythematosus: allergies, infections, and family history. J Clin Epidemiol. 2002;55(10): 982-989.

5. Buyon JP, Petri MA, Kim MY, et al. The effect of combined estrogen and progesterone hormone replacement therapy on disease activity in systemic lupus erythematosus: a randomized trial. Ann Intern Med. 2005; 142(12 Pt 1):953-962.

6. Hahn BH, Ebling F, Singh RR, Singh RP, Karpouzas G, La Cava A. Cellular and molecular mechanisms of regulation of autoantibody production in lupus. Ann N Y Acad Sci. 2005;1051:433-441.

7. Parks CG, Cooper GS. Occupational exposures and risk of systemic lupus erythematosus: a review of the evidence and exposure assessment methods in population- and clinic-based studies. Lupus. 2006; 15(11):728-736.

8. Wong M, Tsao BP. Current topics in human SLE genetics. Springer Semin Immunopathol. 2006;28(2):97-107.

9. Morawski PA, Bolland S. Expanding the B Cell-Centric View of Systemic Lupus Erythematosus. Trends Immunol. 2017;38(5):373-382.
10. Guidelines for referral and management of systemic lupus erythematosus in adults. American College of Rheumatology Ad Hoc Committee on Systemic Lupus Erythematosus Guidelines. Arthritis Rheum. 1999; 42(9):1785-1796.

11. Bertsias G, Ioannidis JP, Boletis J, et al. EULAR recommendations for the management of systemic lupus erythematosus. Report of a Task Force of the EULAR Standing Committee for International Clinical Studies Including Therapeutics. Ann Rheum Dis. 2008;67(2):195-205.

12. Hahn BH, McMahon MA, Wilkinson A, et al. American College of Rheumatology guidelines for screening, treatment, and management of lupus nephritis. Arthritis Care Res. 2012;64(6):797-808.

13. Cobo-Ibáñez T, Loza-Santamaría E, Pego-Reigosa JM, et al. Efficacy and safety of rituximab in the treatment of non-renal systemic lupus erythematosus: a systematic review. Semin Arthritis Rheum. 2014;44(2):175-185.

14. Wilhelmus S, Bajema IM, Bertsias GK, et al. Lupus nephritis management guidelines compared. Nephrol Dial Transplant. 2016;31(6): 904-913.

15. Akhavan PS, Su J, Lou W, Gladman DD, Urowitz MB, Fortin PR. The early protective effect of hydroxychloroquine on the risk of cumulative damage in patients with systemic lupus erythematosus. $J$ Rheumatol. 2013;40(6):831-841.

16. Sugai DY, Gustafson CJ, De Luca JF, et al. Trends in the outpatient medication management of lupus erythematosus in the United States. J Drugs Dermatol. 2014;13(5):545-552.

17. Poh YJ, Baptista B, D’Cruz DP. Subcutaneous and intravenous belimumab in the treatment of systemic lupus erythematosus: a review of data on subcutaneous and intravenous administration. Expert Rev Clin Immunol. 2017;13(10):925-938.

18. Moore PA, Belvedere O, Orr A, et al. BLyS: member of the tumor necrosis factor family and B lymphocyte stimulator. Science. 1999; 285(5425):260-263.

19. Wu Y, Bressette D, Carrell JA, et al. Tumor necrosis factor (TNF) receptor superfamily member TACI is a high affinity receptor for TNF family members APRIL and BLyS. J Biol Chem. 2000;275(45): 35478-35485.

20. Zhang J, Roschke V, Baker KP, et al. Cutting edge: a role for B lymphocyte stimulator in systemic lupus erythematosus. J Immunol. 2001; 166(1):6-10.

21. Stohl W, Metyas S, Tan SM, et al. B lymphocyte stimulator overexpression in patients with systemic lupus erythematosus: longitudinal observations. Arthritis Rheum. 2003;48(12):3475-3486.

22. Collins CE, Gavin AL, Migone TS, Hilbert DM, Nemazee D, Stohl W. B lymphocyte stimulator (BLyS) isoforms in systemic lupus erythematosus: disease activity correlates better with blood leukocyte BLyS mRNA levels than with plasma BLyS protein levels. Arthritis Res Ther. 2006;8(1):R6.

23. Petri M, Stohl W, Chatham W, et al. Association of plasma B lymphocyte stimulator levels and disease activity in systemic lupus erythematosus. Arthritis Rheum. 2008;58(8):2453-2459.

24. Baker KP, Edwards BM, Main SH, et al. Generation and characterization of LymphoStat-B, a human monoclonal antibody that antagonizes the bioactivities of B lymphocyte stimulator. Arthritis Rheum. 2003;48(11):3253-3265.

25. Halpern WG, Lappin $\mathrm{P}$, Zanardi T, et al. Chronic administration of belimumab, a BLyS antagonist, decreases tissue and peripheral blood B-lymphocyte populations in cynomolgus monkeys: pharmacokinetic, pharmacodynamic, and toxicologic effects. Toxicol Sci. 2006;91(2):586-599.

26. Stohl W, Hiepe F, Latinis KM, et al. Belimumab reduces autoantibodies, normalizes low complement levels, and reduces select B cell populations in patients with systemic lupus erythematosus. Arthritis Rheum. 2012;64(7):2328-2337.

27. van Vollenhoven RF, Petri MA, Cervera R, et al. Belimumab in the treatment of systemic lupus erythematosus: high disease activity predictors of response. Ann Rheum Dis. 2012;71(8):1343-1349. 
28. Manzi S, Sánchez-Guerrero J, Merrill JT, et al. Effects of belimumab, a B lymphocyte stimulator-specific inhibitor, on disease activity across multiple organ domains in patients with systemic lupus erythematosus: combined results from two phase III trials. Ann Rheum Dis. 2012; 71(11):1833-1838.

29. Fanouriakis A, Adamichou C, Koutsoviti S, et al. Low disease activityirrespective of serologic status at baseline-associated with reduction of corticosteroid dose and number of flares in patients with systemic lupus erythematosus treated with belimumab: A real-life observational study. Semin Arthritis Rheum. Epub 2018 Feb 23.

30. Furie R, Stohl W, Ginzler EM, et al. Biologic activity and safety of belimumab, a neutralizing anti-B-lymphocyte stimulator (BLyS) monoclonal antibody: a phase I trial in patients with systemic lupus erythematosus. Arthritis Res Ther. 2008;10(5):R109.

31. Wallace DJ, Stohl W, Furie RA, et al. A phase II, randomized, double-blind, placebo-controlled, dose-ranging study of belimumab in patients with active systemic lupus erythematosus. Arthritis Rheum. 2009;61(9):1168-1178.

32. Navarra SV, Guzmán RM, Gallacher AE, et al. Efficacy and safety of belimumab in patients with active systemic lupus erythematosus: a randomised, placebo-controlled, phase 3 trial. Lancet. 2011; 377(9767):721-731.

33. Furie R, Petri M, Zamani O, et al. A phase III, randomized, placebocontrolled study of belimumab, a monoclonal antibody that inhibits $\mathrm{B}$ lymphocyte stimulator, in patients with systemic lupus erythematosus. Arthritis Rheum. 2011;63(12):3918-3930.

34. Ginzler EM, Wallace DJ, Merrill JT, et al. Disease control and safety of belimumab plus standard therapy over 7 years in patients with systemic lupus erythematosus. J Rheumatol. 2014;41(2):300-309.

35. Zhang F, Bae SC, Bass D, et al. A pivotal phase III, randomised, placebo-controlled study of belimumab in patients with systemic lupus erythematosus located in China, Japan and South Korea. Ann Rheum Dis. 2018;77(3):355-363.

36. Pascoe K, Lobosco S, Bell D, et al. Patient- and Physician-reported Satisfaction With Systemic Lupus Erythematosus Treatment in US Clinical Practice. Clin Ther. 2017;39(9):1811-1826.

37. Cai WW, Fiscella M, Chen C, Zhong ZJ, Freimuth WW, Subich DC. Bioavailability, Pharmacokinetics, and Safety of Belimumab Administered Subcutaneously in Healthy Subjects. Clin Pharmacol Drug Dev 2013;2(4):349-357.

38. Shida Y, Takahashi N, Sakamoto T, Ino H, Endo A, Hirama T. The pharmacokinetics and safety profiles of belimumab after single subcutaneous and intravenous doses in healthy Japanese volunteers. J Clin Pharm Ther. 2014;39(1):97-101.
39. Yapa SW, Roth D, Gordon D, Struemper H. Comparison of intravenous and subcutaneous exposure supporting dose selection of subcutaneous belimumab systemic lupus erythematosus Phase 3 program. Lupus. 2016;25(13):1448-1455.

40. Struemper H, Murtaugh T, Gilbert J, et al. Relative Bioavailability of a Single Dose of Belimumab Administered Subcutaneously by Prefilled Syringe or Autoinjector in Healthy Subjects. Clin Pharmacol Drug Dev. 2016;5(3):208-215.

41. Sheikh SZ, Hammer AE, Fox NL, et al. Evaluation of a novel autoinjector for subcutaneous self-administration of belimumab in systemic lupus erythematosus . Int J Clin Pharmacol Ther. 2016;54(11):914-922.

42. Struemper H, Thapar M, Roth D. Population Pharmacokinetic and Pharmacodynamic Analysis of Belimumab Administered Subcutaneously in Healthy Volunteers and Patients with Systemic Lupus Erythematosus. Clin Pharmacokinet. 2018;57(6):717-728.

43. Stohl W, Schwarting A, Okada M, et al. Efficacy and Safety of Subcutaneous Belimumab in Systemic Lupus Erythematosus: A Fifty-TwoWeek Randomized, Double-Blind, Placebo-Controlled Study. Arthritis Rheumatol. 2017;69(5):1016-1027.

44. Doria A, Stohl W, Schwarting A, et al. Efficacy and Safety of Subcutaneous Belimumab in Anti-Double-Stranded DNA-Positive, Hypocomplementemic Patients With Systemic Lupus Erythematosus. Arthritis Rheumatol. 2018;70(8):1256-1264.

45. Doria A, Bass D, Schwarting A, et al. A 6-month open-label extension study of the safety and efficacy of subcutaneous belimumab in patients with systemic lupus erythematosus. Lupus. 2018;27(9):1489-1498.

46. Ramachandran S, Parks D, Kurtinecz M, Roth DA, Alfonso-Cristancho R. An indirect comparison of intravenous and subcutaneous belimumab efficacy in patients with SLE and high disease activity. J Comp Eff Res. 2018;7(6):581-593.

47. Benlysta ${ }^{\circledR}$ (belimumab) [prescribing information]. Rockville (MD): GlaxoSmithKline Ltd; 2017.

48. Falanga M, Canzona A, Mazzoni D. Preference for Subcutaneous Injection or Intravenous Infusion of Biological Therapy Among Italian Patients With SLE. J Patient Exp. 2018;237437351877081.

49. Dashiell-Aje E, Harding G, Pascoe K, Devries J, Berry P, Ramachandran S Patient Evaluation of Satisfaction and Outcomes with an Autoinjector for Self-Administration of Subcutaneous Belimumab in Patients with Systemic Lupus Erythematosus. Patient. 2018;11(1):119-129.
Patient Preference and Adherence

\section{Publish your work in this journal}

Patient Preference and Adherence is an international, peer-reviewed, open access journal that focuses on the growing importance of patient preference and adherence throughout the therapeutic continuum. Patient satisfaction, acceptability, quality of life, compliance, persistence and their role in developing new therapeutic modalities and compounds to optimize

\section{Dovepress}

clinical outcomes for existing disease states are major areas of interest for the journal. This journal has been accepted for indexing on PubMed Central. The manuscript management system is completely online and includes a very quick and fair peer-review system, which is all easy to use. Visit http://www. dovepress.com/testimonials.php to read real quotes from published authors. 